\title{
Identification and tissue distribution of a novel rat glucocorticoid receptor splice variant
}

\author{
Huiming Ju${ }^{1,2}$, Xia Wang ${ }^{3}$, Zongping $\mathrm{Liu}^{2}$, Ping Liu ${ }^{1}$, Yan Zhao ${ }^{1}$, Renping Xiong ${ }^{1}$, \\ Yuanguo Zhou ${ }^{1}$ and Xingyun Chen ${ }^{1 凶}$ \\ ${ }^{1}$ Molecular Biology Center, State Key Laboratory of Trauma, Burn and Combined Injury, Research Institute of \\ Surgery and Daping Hospital, Third Military Medical University, Chongqing, China; ${ }^{2}$ College of Veterinary \\ Medicine, Yangzhou University, Yangzhou, China; ${ }^{3}$ Department of Laboratory Medicine, Western China Hospi- \\ tal, Sichuan University, Chengdu, China
}

Received: 30 October, 2008; revised: 23 February, 2009; accepted: 11 March, 2009 available on-line: 14 March, 2009

\begin{abstract}
Glucocorticoid receptor (GR) is a steroid hormone receptor that has been shown to play important roles in diverse cellular and physiological processes. More and more evidence has revealed that the effects of glucocorticoids are mediated by the glucocorticoid receptor through genomic or nongenomic mechanisms. A growing number of glucocorticoid receptor splice variants have been identified in human tissues, but few are known in rat tissues. In this work, a novel rGR cDNA, called rGR $\beta$, was cloned from Sprague Dawlay (SD) rat liver. Sequence analysis revealed that the rGR $\beta$ mRNA was 39 base pairs (bp) shorter than the rGR mRNA reported earlier. The deleted segment is located in exon 1 and encodes 13 repeated glutamine residues. Both the rGR and rGR $\beta$ mRNAs were quantitated by Northern blot hybridization using non-homologous glucocorticoid cDNA probes. Results showed that the rGR and rGR $\beta$ mRNAs were most abundant in the lung, the least abundant in the heart, and there were more rGR and rGR $\beta$ mRNAs in the kidney than in the liver. The identification of $\operatorname{rGR} \beta$ may contribute to the understanding of the genomic or nongenomic effects of glucocorticoids.
\end{abstract}

Keywords: glucocorticoid receptor, splice variant, receptor isoform, glucocorticoid

\section{INTRODUCTION}

Glucocorticoid receptor (GR), which belongs to the large nuclear receptor family as an important endocrine signaling molecule, controls reproduction, development, metabolism, salt balance and specialized cellular responses, such as inflammation and immunity (Barnes \& Adcock, 2003). GR is present in the cytoplasm as a multiprotein complex containing (co)chaperones like heat shock proteins (HSPs) and immunophilins (Pratt et al., 2004). After binding with glucocorticoids (GCs) in the cytoplasm, the activated receptor is translocated into the nucleus and subsequently binds to negative or positive GR responsive elements (GREs) in the promoter regions of target genes (Franchimont, 2004), resulting in the enhancement or inhibition of mRNA and protein synthesis (Yamamoto, 1985; Ito et al., 2006). This theory, which is also called the genomic effects of GCs, is the underlying mechanism for the use of GCs in the treatment of allergic and autoimmune disorders (Keller-Wood \& Dallamn, 1984; Scadding, 2001; Busse et al., 2002). In recent years, it has been suggested that many effects of glucocorticoids were mediated by a nongenomic mechanism through classic glucocorticoid receptors (HafeziMoghadam et al., 2002; Lowenberg et al., 2005).

${ }^{\square}$ Corresponding author: Xingyun Chen, Molecular Biology Center, State Key Laboratory of Trauma, Burn and Combined Injury, Research Institute of Surgery and Daping Hospital, Third Military Medical University, Chongqing, China, 400042; tel.: +86023 6875 7472; fax: +86023 6881 7159; e-mail: xingyunchen@gmail.com

Abbreviations: GAPDH, glyceraldehyde phosphate dehydrogenase; GC, glucocorticoid; GR, glucocorticoid receptor; GRE, glucocorticoid receptor responsive element; PAGE, polyacrylamide gel electrophoresis; SDS, sodium dodecyl sulfate. 
The GR gene is located on chromosome 18 and consists of 8 exons and 7 introns spanning more than $60 \mathrm{~kb}$ of the rat genome, with protein coding beginning from exon 1. Two different isoforms of GR, GR $\alpha$ and GR $\beta$, which are derived from alternative splicing of the GR primary mRNA, have been identified in humans. GR $\alpha$ plays an important role in the genomic effect of glucocorticoids, while GR $\beta$ has a dominant negative effect on GR $\alpha$ through the formation of GR $\alpha / G R \beta$ heterodimers (Bamberger et al., 1995; Oakley et al., 1997). The structures and functions of human GR $\alpha$ and GR $\beta$ have been reviewed in several articles (Pujols et al., 2004; 2007; Rhen \& Cidlowski, 2005).

In the 1980s, the intracellular rat glucocorticoid receptor (rGR) was identified (Yamamoto, 1985; Miesfeld et al., 1986). It was reported that the strength of the transcription activation correlates with the length of the protein (Lavery \& McEwan, 2005). Gearing et al. (1993) reported that the Sprague Dawlay rat genomic DNA gave 2 rGR PCR products that encoded either 7 or 17 glutamines at this position, which was different from the 19 glutamines of the published rGR sequence. Until now, no rGR splice variant has been described and the function of the rGR isoform is unclear, A novel rGR cDNA (Ensembl accession number, ENSRNOT00000044287) was predicted by the Ensembl automatic analysis pipeline using a GeneWise/Exonerate model from a protein database or a set of aligned cDNAs followed by an ORF prediction (named predicted rGR $\beta$ ). In this work, we identified and cloned a new rGR mRNA splice variant, rGR $\beta$. In addition, we examined the distribution of rGR $\beta$ and the early reported rGR mRNA in main tissues of rats. Our identification of a new rGR variant may contribute to the understanding of the genomic and nongenomic actions of glucocorticoids mediated by classic glucocorticoid receptors. It is possible that the genomic and nongenomic effects of glucocorticoids are mediated by different splice variants of the same glucocorticoid receptor.

\section{MATERIALS AND METHODS}

Animals. Adult Sprague Dawlay rats (male and female) weighing 180-220 g were used in all experiments. Animals were maintained in groups of 2 3 under controlled temperature $\left(25^{\circ} \mathrm{C}\right)$ and lighting conditions (12:12-hour light-dark cycle) and with free access to rat chow and water. The procedures of the experiment and animal handling practices were approved by the Animal Care Committee of the Third Military Medical University.

Cloning of rGR $\beta$ cDNA. The rats were anesthetized and sacrificed. Total RNA was extracted from the rat liver using Trizol according to the manufacturer's protocol (Invitrogen, Carlsbad, CA, USA). The $A B$ domain fragment of GR was synthesized by RT-PCR with total RNA using one step RT-PCR following the manufacturer's instructions (TaKaRa, Shiga, Japan; Product \# DRR018A). The initial PCR program used was $94^{\circ} \mathrm{C}$ for $5 \mathrm{~min}, 94^{\circ} \mathrm{C}$ for $30 \mathrm{~s}$, $60^{\circ} \mathrm{C}$ for $30 \mathrm{~s}$, and $72^{\circ} \mathrm{C}$ for $1 \mathrm{~min}$, repeat steps $2-4$ for 39 cycles, then $72^{\circ} \mathrm{C}$ for $10 \mathrm{~min}$. This reaction utilized a GR-AB sense primer (nucleotide, nt 66), 5'-ataGAATTCCCAATGGACTCCAAAGAATC-3', with the anti-sense primer (nt 1427), 5'-aatGGATCCCCCGTAATGACATCCTGAAG-3'. The restriction enzyme sites for EcoRI and BamHI were incorporated into the forward and reverse primers, respectively. Purified PCR products were ligated into the pGADT7 vector, which was used to transform the E. coli $\mathrm{DH} 5 \alpha$ cells. The positive colonies were selected for the preparation of plasmid DNA using a crude alkaline lysis protocol. Purified plasmid DNA was prepared for sequencing, and the obtained sequences were aligned with Genbank database by BLAST. The plasmid with the novel rGR was named pGADT7-GR $\beta$.

Probe preparation. The cDNA probes were synthesized by Nest-PCR, with rat genomic DNA and pGADT7-GR $\beta$ as the templates. For these reactions, two pairs of primers were utilized. The outer pair was the sense primer 5'-ACGAAGTCCCTGGCAGTTTG-3' and antisense primer 5'-CAGTTGCAGACGTTGAACTC-3'. The inner pair of primers was sense primer 5'-CAATGTGCAGCAGCGACAG$3^{\prime}$ and antisense primer 5'-GGACAGTGAAACGGCTTTG-3'. The $5^{\prime}$ end of the inner pair of primers was labeled with biotin. The PCR program used was $94^{\circ} \mathrm{C}$ for $5 \mathrm{~min}, 94^{\circ} \mathrm{C}$ for $30 \mathrm{~s}, 55^{\circ} \mathrm{C}$ for $30 \mathrm{~s}$, and $72^{\circ} \mathrm{C}$ for $30 \mathrm{~s}$, repeat steps $2-4$ for 34 cycles, then $72^{\circ} \mathrm{C}$ for $5 \mathrm{~min}$. Biotin activity of the two PCR products was measured by Detector ${ }^{\mathrm{TM}}$ AP Chemiluminescent Blotting Kit (KPL, Gaithersburg, MD, USA; Catalog No. 54-30-01). A single stranded probe specific for glyceraldehyde phosphate dehydrogenase (GAPDH) was synthesized by Invitrogen and its 5' end was labeled with biotin (5'-AAGGCCATGCCAGTGAGCTTCCCGTTCA-3'). The specificity of all the probes was verified by dot blot analysis.

RNA isolation and Northern blot analysis. Rat lung, liver, heart and brain were freshly excised, and total RNA was extracted using Trizol according to the procedure described by the manufacturer. mRNA was isolated using the Innogene Kit (Singapore). Samples of isolated mRNA (10 $\mu \mathrm{g} /$ lane) were electrophoresed in $1 \%(\mathrm{w} / \mathrm{v})$ formaldehyde agarose gel, blotted onto a nylon membrane and crosslinked by exposure to UV. Hybridization for Northern blot analysis was performed in $50 \%(\mathrm{v} / \mathrm{v})$ formamide at $42^{\circ} \mathrm{C}$. Membranes were washed in $0.2 \times \mathrm{SSC}, 0.1 \%$ 
$(\mathrm{w} / \mathrm{v})$ SDS at $55^{\circ} \mathrm{C}$, and detected with rGR and rGR $\beta$, respectively, using Detector ${ }^{\mathrm{TM}}$ AP Chemiluminescent Blotting Kit (KPL, Catalog No. 54-30-01). The membranes were placed in a hybridization bag and exposed to an X-ray film for about $5 \mathrm{~min}$. X-Ray film was scanned using a scanner to quantitate the bands. To assure specificity of the results obtained with the GR probe, the membranes were subsequently hybridized with the GAPDH probe. The gray scales for GR and GAPDH were quantitated by Image Pro Plus software (v. 4.5). The gray scale for GR in each sample was normalized with that of GAPDH for minor variations in the amount of RNA loaded in each well.

\section{RESULTS}

\section{Characterization of rGR cDNA and amino-acid sequence of the deduced polypeptide}

The rGR (UniProtKB/Swiss-Prot accession number, P06536) consists of three domains: modulating domain (rGR-AB, aa 1-439), DNA-binding domain (rGR-CD, aa 440-505) and steroid-binding domain (rGR-EF, aa 547-795). The PCR products of the rat genomic DNA amplified with the rGR-AB primer had the same sequence as that of the reported rGR (GenBank accession number, NC_005117, NM_012576). A novel rGR-AB subunit variant, designated as rGR-AB $\beta$, was isolated from rat hepatic tissue and identified by sequencing. The full-length of rGR-AB $\beta$ consisted of $1275 \mathrm{bp}$ (Fig. 1A, rGR-AB $\beta$ ) coding a polypeptide containing 425 amino acids (Fig. 1B, rGR-AB $\beta$ aa). In rGR-AB $\beta$, the $245 \mathrm{nt}$ to 283 nt region was deleted as compared with the reported rGR cDNA sequence. The deletion was located at exon 1, which encoded 13 glutamine $(Q)$ residues.
The cDNA sequence of the rGR-AB $\beta$ and deduced polypeptide aligned with the sequence of $\mathrm{rGR}$ and their sequences along with the predicted rGR $\beta$ sequence (ENSRNOT00000044287) are shown in Fig. 1.

\section{Preparation of the rGR and rGR $\beta$ probes by PCR}

The preparation of rGR and rGR $\beta$ probes were carried out by Nest-PCR with rat genomic DNA and pGADT7-GR $\beta$. Separation of the PCR products by polyacrylamide gel electrophoresis (PAGE) showed that the lengths of the products were consistent with the sequencing data. The size of PCR products with rat genomic DNA as the template was approx. 100 $\mathrm{bp}$, and the size of the PCR products with pGADT7GR $\beta$ as the template was approx. $60 \mathrm{bp}$. When the cDNA of rat liver was used as the template, there were two PCR products detected by agarose gel electrophoresis. This result suggested that there were two splice variants at the cDNA level (Fig. 2).

\section{Distribution of rGR and rGR $\beta$ mRNAs in tissues}

Poly(A)-enriched mRNA derived from heart, liver, lung and kidney of normal Sprague Dawlay rats was denatured, separated by agarose gel electrophoresis, and detected by hybridizing with rGR and rGR $\beta$ probes. As shown in Fig. 3, a single broad band of $5-7.0 \mathrm{~kb}$ corresponding to glucocorticoid mRNA and a single band about $2.0 \mathrm{~kb}$ corresponding to GAPDH mRNA was identified in all tissues. Densitometric scanning of the autoradiographs indicated that rGR and rGR $\beta$ mRNAs were most abundant in the lung (Fig. 3D). The rGR mRNA levels in the kidney, liver and heart were $58 \%, 38 \%$, and $10 \%$ of that in the lung, respectively. The rGR $\beta$ mRNA levels in the kidney, liver and heart were $89 \%, 38 \%$, and $18 \%$ of that in the lung, respectively.

rGR genomic DNA 819 age at gtg cag cag cga cag cag cag cag cag cag cag cag cag cag cag cag cag cag cag

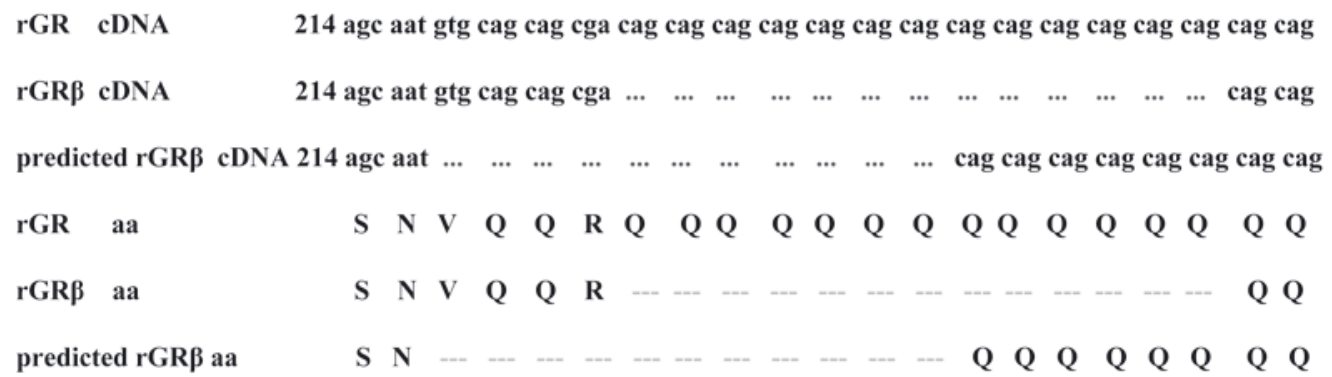

Figure 1. Nucleotide and deduced amino-acid sequences alignment of the rGR genomic DNA, rGR cDNA, rGR $\beta$ and predicted $\mathrm{rGR} \beta \mathrm{cDNAs}$

Ensembl accession number, ENSRNOT00000044287). The deletion in rGR-AB $\beta$, rGR-AB $\beta$ and predicted rGR $\beta$ amino-acid sequence was represented by dotted lines. The nucleotide and amino-acid sequence of rGR is from GenBank (GenBank accession number, NM 012576, NC 005117). The nucleotide sequence of rGR-AB $\beta$ has been deposited into the GenBank database under the accession number EF495208. 


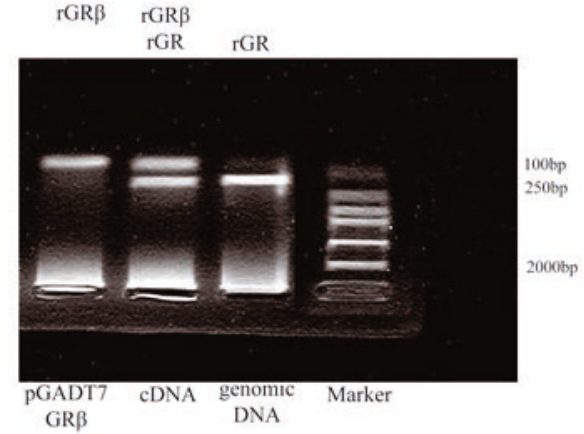

Figure 2. Electrophoresis of the rGR and $\operatorname{rGR} \beta$ probes on agarose gel.

Nest-PCR products separated on a $2.5 \%$ agarose gel is shown. The Marker lane was the DNA marker DL2, 000 (Takara, Catalog No. D501A). PCR was carried out with plasmid pGADT7-GR $\beta$ (lane pGADT7-GR $\beta$ ), rat cDNA of liver (lane cDNA) and genomic DNA (lane genomic DNA). There were two products in lane cDNA (rGR and rGR $\beta$ ) and only one product in lane pGADT7-GR $\beta$ (rGR $\beta$ ) or lane genomic DNA (rGR).

\section{DISCUSSION}

The difference between the sequences of the rGR $\beta$ and reported rGR cDNA lies at the modulating domain of rGR, which is structurally flexible and contains surfaces involved in both the activation and repression of gene transcription. We obtained one rGR PCR product with rat genomic DNA as the template and two PCR products with rat cDNA as the template, indicating the existence of splice variants as predicted. However, there were some differences between the predicted rGR $\beta$ and the rGR $\beta$ that was cloned by us (Fig. 1). The function of the modulating domain of rGR is still unclear, but there is evidence that it might be important in mediating transcriptional repression by rGR (Iniguez-Lluhi et al., 1997). Many transcription factors contain glutamine rich regions in their activation domains (Mitchell \& Tjian, 1989). The length of the glutamine region may affect the transcriptional activity. Based on this assumption, the biological effect of rGR $\beta$ may be different from rGR, one of the splice variants may be involved in the genomic effects and the other may be involved in the nongenomic effects of glucocorticoids.

We used Northern blot analysis to examine the distribution of rGR and rGR $\beta$ mRNA in normal rat tissues. Most of the early studies of rGR with a rGR probe could not distinguish the two rGR mRNAs effectively (Kalinyak et al., 1987; Levy et al., 1989; Kitraki et al., 1999; Pfeiffer \& Barden, 1988). In our study, we designed two different probes based on their non-homologous region. The specificity of the probes was verified by dot blot analysis. Hybridization results showed that the probes can distinguish rat genomic DNA and pGADT7-GR $\beta$. A major transcript of approx. $7-\mathrm{kb}$ was detected in
(A)

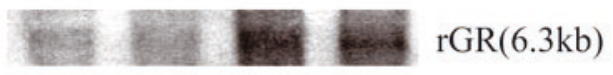

(B)

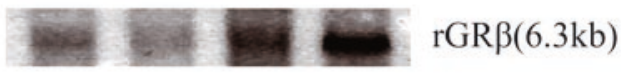

(C)

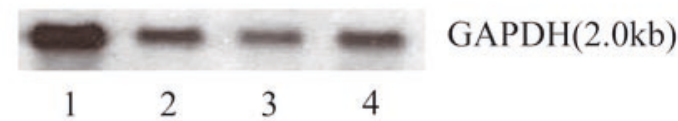

(D)

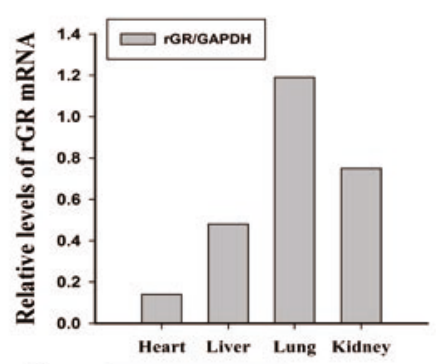

(E)

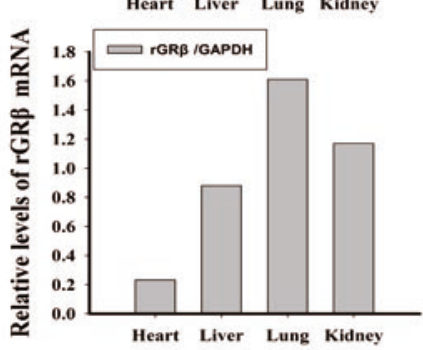

Figure 3. Northern blot analysis of rGR and $\operatorname{rGR} \beta$ mRNA in different rat tissues.

Poly(A)-enriched mRNA (10 $\mu \mathrm{g}$ per lane) obtained from heart (lane 1), liver (lane 2), lung (lane 3) and kidney (lane 4) of normal rats was denatured with glyoxal and separated on $1 \%$ formaldehyde agarose gel. The mRNA was transferred to a nylon membrane and hybridized with rGR, rGR $\beta$, and GAPDH probes, separately. A. Hybridization with rGR probe. B. Hybridization with rGR $\beta$ probe. C. Hybridization with rat GAPDH oligonucleotide probe. D. A plot of the relative levels of rGR mRNA in different rat tissues. E. A plot of the relative levels of rGR $\beta$ mRNA in different rat tissues.

rat tissues. We found that both the rGR and rGR $\beta$ mRNA were most abundant in the lung. The higher levels of rGR and rGR $\beta$ mRNAs in the kidney than in the liver suggested that the total rGR mRNA in kidney was more than that in the liver, which was different from the early report (Kalinyak et al., 1987). The probe used in their experiments was located at the 3' nontranslated region (Miesfeld et al., 1984; Kalinyak et al., 1987), which could not distinguish the rGR and rGR $\beta$ mRNA. Up to now, it is still unclear whether the different distribution of the two types of rGR in tissues is related to their functions in different tissues.

The traditional GR receptor blocking agents, such as RU486, mainly exert their antiglucocorticoid activity through the prevention of complete 
GR transformation and alteration of the steps after GR-DNA binding (Mao et al., 1992; Beck et al., 1993). It is unclear whether RU486 can interact with the $\mathrm{N}$-terminal domain of GR and block its function. It would be interesting to identify ways to specifically modify the actions of rGR and rGR $\beta$ and study their specific biological functions. Specific antibody, RNA interference, or some specific agents could be used for this purpose.

In conclusion, we showed that the novel rGR mRNA splicing occurred at different degrees in different rat tissues. Our identification of a novel splice variant of rGR could aid the further study of the genomic and nongenomic effects of GCs-mediated by rGR or rGR $\beta$.

\section{Acknowledgements}

This work was supported by National Natural Science Foundation of China (X.Y.C., 30300423), Foundation for the Author of National Excellent Doctoral Dissertation of P.R. China (Y.G.Z., 200156) and Natural Science Foundation Project of CQ CSTC(CSTC, 2006BB5105).

\section{REFERENCES}

Bamberger CM, Bamberger AM, de Castro M, Chrousos GP (1995) Glucocorticoid receptor beta, a potential endogenous inhibitor of glucocorticoid action in humans. J Clin Invest 95: 2435-2441.

Barnes PJ, Adcock IM (2003) How do corticosteroids work in asthma? Ann Intern Med 139: 359-370.

Beck CA, Estes PA, Bona BJ, Muro-Cacho CA, Nordeen SK, Edwards DP (1993) The steroid antagonist RU486 exerts different effects on the glucocorticoid and progesterone receptors. Endocrinology 133: 728-740.

Busse WW, Lenfant C, Lemanske RF Jr (2002) Asthma guidelines: a changing paradigm to improve asthma care. J Allergy Clin Immunol 110: 703-705.

Franchimont D (2004) Overview of the actions of glucocorticoids on the immune response: a good model to characterize new pathways of immunosuppression for new treatment strategies. Ann NY Acad Sci USA 1024: 124-137.

Gearing KL, Gustafsson JA, Okret S (1993) Heterogeneity in the polyglutamine tract of the glucocorticoid receptor from different rat strains. Nucleic Acids Res 21: 2014.

Hafezi-Moghadam A, Simoncini T, Yang Z, Limbourg FP, Plumier JC, Rebsamen MC, Hsieh CM, Chui DS, Thomas KL, Prorock AJ (2002) Acute cardiovascular protective effects of corticosteroids are mediated by nontranscriptional activation of endothelial nitric oxide synthase. Nat Med 8: 473-479.

Iniguez-Lluhi JA, Lou DY, Yamamoto KR (1997) Three amino acid substitutions selectively disrupt the activation but not the repression function of the glucocorticoid receptor N terminus. J Biol Chem 272: 4149-4156.
Ito K, Chung KF, Adcock IM (2006) Update on glucocorticoid action and resistance. J Allergy Clin Immunol 117: 522-543.

Kalinyak JE, Dorin RI, Hoffman AR, Perlman AJ (1987) Tissue specific regulation of glucocorticoid receptor mRNA by dexamethasone. J Biol Chem 262: 1044110444.

Keller-Wood ME, Dallman MF (1984) Corticosteroid inhibition of ACTH secretion. Endocr Rev 5: 1-24.

Kitraki E, Karandrea D, Kittas C (1999) Long-lasting effects of stress on glucocorticoid receptor gene expression in the rat brain. Neuroendocrinology 69: 331-338.

Lavery DN, McEwan IJ (2005) Structure and function of steroid receptor AF1 transactivation domains: induction of active conformations. Biochem J 391: 449-464.

Levy FO, Ree AH, Eikvar L, Govindan MV, Jahnsen T, Hansson V (1989) Glucocorticoid receptors and glucocorticoid effects in rat Sertoli cells. Endocrinology 124: 430-436.

Lowenberg M, Tuynman J, Bilderbeek J, Gaber T, Buttgereit F, van Deventer S, Peppelenbosch M, Hommes D (2005) Rapid immunosuppressive effects of glucocorticoids mediated through Lck and Fyn. Blood 106: 1703-1710.

Mao J, Regelson W, Kalimi M (1992) Molecular mechanism of RU 486 action: a review. Mol Cell Biochem 109: 1-8.

Miesfeld R, Okret S, Wikstrom AC, Wrange O, Gustafsson JA, Yamamoto KR (1984) Characterization of a steroid hormone receptor gene and mRNA in wild-type and mutant cells. Nature 312: 779-781.

Miesfeld R, Rusconi S, Godowski PJ, Maler BA, Okret S, Wikstrom AC, Gustafsson JA, Yamamoto KR (1986) Genetic complementation of a glucocorticoid receptor deficiency by expression of cloned receptor cDNA. Cell 46: 389-399.

Mitchell PJ, Tjian R (1989) Transcriptional regulation in mammalian cells by sequence-specific DNA binding proteins. Science 245: 371-378.

Oakley RH, Webster JC, Sar M, Parker CR Jr, Cidlowski JA (1997) Expression and subcellular distribution of the $\beta$-isoform of the human glucocorticoid receptor. Endocrinology 138: 5028-5038.

Pfeiffer A, Barden N (1988) Glucocorticoid receptor gene expression in rat pituitary gland intermediate lobe following ovariectomy. Mol Cell Endocrinol 55: 115-120.

Pratt WB, Galigniana MD, Morishima Y, Murphy PJ (2004) Role of molecular chaperones in steroid receptor action. Essays Biochem 40: 41-58.

Pujols L, Mullol J, Picado C (2007) Alpha and beta glucocorticoid receptors: relevance in airway diseases. Curr Allergy Asthma Rep 7: 93-99.

Pujols L, Mullol J, Torrego A, Picado C (2004) Glucocorticoid receptors in human airways. Allergy 59: 10421052.

Rhen T, Cidlowski JA (2005) Antiinflammatory action of glucocorticoids - new mechanisms for old drugs. $N$ Engl J Med 353: 1711-1723.

Scadding GK (2001) Corticosteroids in the treatment of pediatric allergic rhinitis. J Allergy Clin Immunol 108: S59-64.

Yamamoto KR (1985) Steroid receptor regulated transcription of specific genes and gene networks. Annu Rev Genet 19: 209-252. 